\title{
Real-time Adaptive Parameter Estimation for a Polymer Electrolyte Membrane Fuel Cell
}

\author{
Yashan Xing, Jing Na, Member, IEEE, and Ramon Costa-Castelló, Senior Member, IEEE
}

\begin{abstract}
In this paper we propose real-time adaptive parameter estimation methods for a polymer electrolyte membrane fuel cell (PEMFC) to facilitate the modeling and the subsequent control synthesis. Specifically, the electrochemical model of this fuel cell is in a nonlinearly parametric formulation. Hence, most of existing parameter estimation techniques for PEMFC mainly rely on the optimization approaches, requiring heavy computational costs or even offline implementation. In comparison to those methods, real-time adaptive parameter estimation methods for nonlinearly parametric system are developed in this paper. First, the nonlinearly parametric function is linearized by using the Taylor series expansion. Then, adaptive parameter estimation methods are proposed for estimating the constant or time-varying parameters of PEMFC. Different from the wellrecognized adaptive parameter estimation methods, the proposed adaptive laws are driven by the extracted estimation errors, so that exponential convergence of the parameter estimation error can be guaranteed, without using any predictors or observers. Finally, practical experiments in a H-100 PEMFC system are conducted, which illustrate satisfactory performances of the presented parameter estimation methods under different operation scenarios.
\end{abstract}

Index Terms-Adaptive parameter estimation, nonlinearly parametric system, polymer electrolyte membrane fuel cell, timevarying parameters.

\section{INTRODUCTION}

$\mathbf{F}$ UEL cells as sustainable energy conversion devices have already attracted considerable interests of both academia and engineers in recent years [1]. They can directly convert hydrogen and oxygen into water, thermal energy and electricity through the electrochemical reaction. Based on variety of electrolyte materials, there are different types of fuel cells, such as solid oxide fuel cell (SOFC), polymer electrolyte membrane fuel cell (PEMFC), and alkaline fuel cell (AFC), etc.

Polymer electrolyte membrane fuel cells (PEMFCs) have been recognized as one of the most promising devices for commercial application due to their specific advantages, such

This work was partially supported by the National Natural Science Foundation of China under Grant 61873115, 61573174, the Spanish national project MICAPEM (ref. DPI2015- 69286-C3-2-R, MINECO/FEDER), the Spanish State Research Agency through the María de Maeztu Seal of Excellence to IRI (MDM-2016-0656), AGAUR of Generalitat de Catalunya through the Advanced Control Systems (SAC) group grant (2017 SGR 482), and Chinese Scholarship Council (CSC) under grant (201808390007). (Corresponding author: Jing $\mathrm{Na}$ ).

Jing $\mathrm{Na}$ is with the Faculty of Mechanical and Electrical Engineering, Kunming University of Science and Technology, Kunming, 650500, P.R. China. (Email: najing25@163.com)

Yashan Xing and Ramon Costa Castelló are with Institut de Robòtica i Informàtica Industrial, CSIC-UPC. C/ Llorens i Artigas 4-6, 08028 Barcelona, Spain.(Email: yashan.xing@outlook.com,ramon.costa@upc.edu) as low operation temperature, zero pollutant emission, high efficiency and flexible installation, etc [2]. However, PEMFCs have not been widespread used since the stack degradation may cause low durability and high life-cycle costs [3]. In this respect, many studies on the modeling of PEMFCs have been carried out for the purpose of analyzing, optimizing and monitoring their operation performance. In brief, the models of PEMFCs can be generally divided into three types: i) mechanistic models range zero-dimensional to three-dimensional models, which are based on the material property, physical structure and electrochemical phenomena [4]-[6]; ii) datadriven models, which are highly dependent on experimental data [7]; iii) semi-empirical models, which consider the combination of physical and empirical models [8]. Since PEMFC is a highly nonlinear system and some parameters are related to the operation condition, these models mentioned above are still difficult to accurately predict the PEMFC characteristics. Thus, it is necessary to exploit parameter estimation methods for PEMFC to online determine unknown model parameters by using easily measurable input and output data.

Adaptive parameter estimation has been widely studied during the past decades. In general, the well-known adaptive methods are designed to handle the parameter estimation by minimizing the observer error based on the gradient descent algorithm [9], least square (LS) method [10], or even neural network [11], [12], etc. However, most of these parameter estimation methods have been developed to estimate constant parameters only, and assuming the unknown parameters are in the linearly parametric systems. These stringent assumptions may not be true for fuel cell systems. In [13], recursive least square (LS) algorithms were employed for parameter estimation of PEMFC based on a semi-empirical model, where the unknown time-varying parameters are assumed to be independent of the regressor matrix. However, estimating timevarying parameters still remains as an open problem [14].

In fact, PEMFC is a typical nonlinearly parametric system, where some of unknown parameters, especially time-varying parameters, are embedded in nonlinear functions. However, only few studies have been reported on the real-time parameter estimation of nonlinearly parametric systems. For instance, an adaptive estimation method based on the convexity or concavity parametrization and the min-max algorithm was proposed in [15], where certain assumptions that are not true for fuel cell plants are imposed. Hence, most of the parameter estimation schemes for PEMFC have been designed based on intelligent optimization techniques. In [16]-[18], genetic algorithms (GA) were applied into curve fitting procedures to handle the PEMFC parameter identification problem. However, genetic 
algorithms have inherent limitations in terms of searching efficiency, i.e. premature convergence, highly correlated parameters, etc [19]. In order to avoid these degradations in GA schemes, particle swarm optimization (PSO) was employed to estimate PEMFC parameters based on experimental data in [20]. Compared with traditional optimization methods and GA approaches, the PSO can achieve better estimation results. More recently, evolution algorithm [21] was also used to estimate parameters based on the circuital model of PEMFC. However, all of above mentioned optimization methods cannot address the estimation problem online since they focus exclusively on offline searching procedures, which introduce heavy computational costs in the practical implementation and thus cannot be used in the real-time applications.

This paper aims to exploit real-time adaptive parameter estimation methods for a nonlinearly parametric PEMFC system. Specifically, the Taylor series expansion is used to extract the unknown parameters from the electrochemical functions. Then, adaptive parameter estimation algorithms are developed to estimate constant or time-varying parameters in the PEMFC model, where the convergence of estimation errors is guaranteed under the persistent excitation (PE) condition. Comparing to other classic estimation approaches relied on the predictor or observer errors with gradient descent algorithms, the main feature of the proposed methods is that the adaptive laws are driven by parameter estimation errors, which help to guarantee fast estimation performance even for nonlinearly parametric time-varying parameters. Experimental validations are carried out based on a practical PEMFC plant to show the feasibility and satisfactory performance of the proposed estimation methods.

The remainder of this paper is organized as follows: In Section II, the electrochemical model of PEMFC is briefly introduced. Section III presents the proposed parameter estimation methods for constant and time-varying parameters. In Section IV and Section V, the experimental setup and comparative results are discussed. Finally, conclusions are provided in Section VI.

\section{The Mathematical Models of PEMFC}

The PEMFC studied in this paper is an open-cathode fuel cell, which has been widely used. In previous works [22], [23], the modeling for this type of PEMFCs has been addressed. In order to determine the parameters to be estimated, a brief description about PEMFC operation principle and its corresponding lumped model is introduced in this section.

\section{A. Operation Principle}

The core of a PEMFC is a polymer membrane that acts as an electrolyte in contact with positive and negative electrodes. Figure 1. shows the schematic diagram of a PEMFC. The electrochemical reaction of PEMFC occurs at the interface between the electrolyte and positive electrode. At the anode, hydrogen as a fuel releases electrons and protons. Then protons pass through the electrolyte layer to the cathode whereas electrons travel through outside circuit to the cathode. They are combined with oxygen at the cathode to produce water

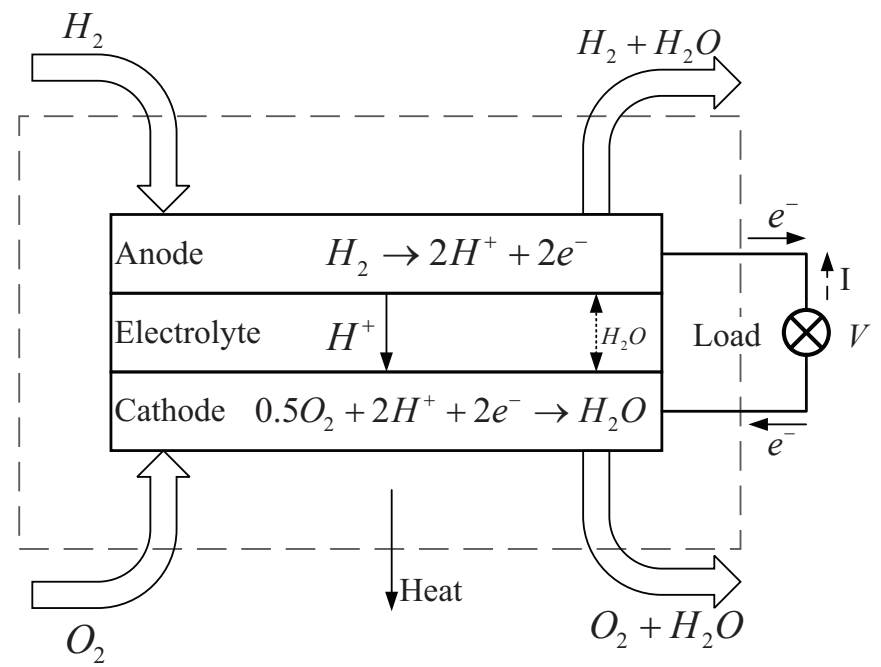

Figure 1. Schematic diagram of a PEMFC.

and heat. The electrolyte has a main function that only allows hydrogen ions to pass. For water, it can go through the electrolyte in both directions as a result of the pressure difference and water concentration in both electrodes. Moreover, the conductivity of the polymer membrane is dependent on its hydration. The resistance can be increased for lack of hydration whereas excessive hydration can limit protons' transport. Thus, water transport can be regarded as a key factor to affect the PEMFC operation performance.

\section{B. Electrochemical Model}

The electrochemical model of PEMFC is used to predict the voltage. In practice, the theoretical maximum cell potential, $E_{r}$, is larger than the actual voltage output, $V$. In PEMFC, there are three major potential losses: ohmic losses, $V_{\text {ohm }}$, activation losses, $V_{a c t, c}$, and concentration losses, $V_{c o n}$, [1]. To be specific, the ohmic loss is derived from electrons and protons traveling from the electrodes and membrane. For the activation loss, it occurs on the interface between the electrodes and electrolyte which is used to drive the electrochemical reaction. The concentration loss is an irreversible loss that happens due to the change of reactant concentration. In this study, the concentration loss is not considered.

Ohmic losses can be described by ohm's law

$$
V_{o h m}=R_{o h m} I
$$

where $I$ is the current; $R_{o h m}$ is the ohmic resistance.

The activation polarization losses happened at the cathode can be expressed as a function of current density, that is

$$
V_{a c t, c}=\frac{R T}{\alpha_{c} n F} \ln \left(\frac{i}{i_{0, c}}\right)
$$

where $i=I / A_{c l}$ is the current density; $A_{c l}$ denotes the surface activation area of catalyst layer; $\alpha_{c}$ is the charge transfer coefficient; $F$ and $R$ are the Faraday's constant and the ideal gas constant, respectively; $n$ is the number of electrons involved in the chemical reaction; $T$ is the stack temperature; 
$i_{0, c}$ denotes the exchange current density that represents the equilibrium rate at the chemical reaction process. It is a very sensitive value in the activation losses which heavily depends on the electrode physical properties and operation conditions. Considering the effect of liquid water saturation, the exchange current density at the cathode is calculated as follows:

$$
i_{0, c}=\gamma_{c} \cdot \operatorname{ECSA}(s) \cdot\left(\frac{p_{\mathrm{O}_{2}}}{p_{\text {ref }}}\right)^{0.25} e^{-\frac{E_{c a}}{R T}\left(1-\frac{T}{T_{r e f}}\right)}
$$

where $\gamma_{c}$ is the exchange current density constant at the ambient condition $\left(T_{\text {ref }}=293 \mathrm{~K}\right.$, and $p_{\text {ref }}=1$ atm); $p_{\mathrm{O}_{2}}$ represents the pressure of oxygen; $E_{c a}$ is the activation energy for oxygen reaction at the cathode catalyst layer; The electrochemical active surface area, $E C S A$, is heavily related to the liquid water saturation, $s$, which is difficult to measure in the experiments. The empirical expression of ECSA in [22] is written as

$$
\operatorname{ECSA}(s)=A_{0}\left(e^{K_{c} s}-1\right)
$$

where $A_{0}$ is the pre-exponential constant of electrochemical active area; $K_{c}$ is the active area reduction rate.

Based on the activation polarization and ohmic losses defined in (1)-(2), the voltage balance is calculated as

$$
V=n_{\text {cell }}\left(E_{r}-V_{o h m}-V_{a c t, c}\right)
$$

where $E_{r}$ is the theoretical voltage; $n_{\text {cell }}$ represents the number of cells in a PEMFC stack.

\section{Problem Formulation}

In (4), the function of the electrochemical active surface area, $E C S A$, is the empirical expression that is explored in [24]. This parameter is highly dependent on the time-varying water saturation, $s$, which is very difficult to measure and has relevant impact on the voltage of PEMFC. Moreover, the ohmic resistance, $R_{o h m}$, is considered as a constant in some models [6]. However, the ohmic resistant is related to the operation temperature and the water transport in practice. Thus, the parameters to be estimated in this paper are selected as:

$$
\theta=\left[\begin{array}{ll}
R_{o h m} & \frac{1}{E C S A}
\end{array}\right]^{\mathrm{T}}
$$

Since the parameter, $E C S A$, in (3) is embedded in the natural logarithmic function of (2), we take $\theta_{2}=1 / E C S A$ as the reciprocal form of parameter $E C S A$ in (6), which avoids the complexity in the following mathematical developments. Hence, the equation (5) can be formulated as follows:

$$
\begin{aligned}
V & =n_{\text {cell }}\left[E_{r}-V_{\text {ohm }}\left(I, R_{o h m}\right)-V_{\text {act }, c}(I, T, E C S A)\right] \\
& =V(I, T, \theta)
\end{aligned}
$$

As shown in (7), the parameters to be estimated in the electrochemical model are in a nonlinearly parametric form, i.e. the unknown parameter $\theta$ is embedded in the nonlinear function $V(I, T, \theta)$. This makes most of existing parameter estimation schemes invalid for (7), since they have mainly been developed for linearly parametric systems with $\theta$ not being included in the regressor function.

Hence, the objective is to propose real-time adaptive parameter estimation algorithms for nonlinearly parametric PEMFC model (7), which allows to estimate the unknown parameters of the ohmic resistance $R_{o h m}$ and the electrochemical active surface area $E C S A$ through using the measurable variables (i.e. current $I$, temperature $T$, and voltage $V$ ).

\section{Parameter Estimation Algorithm}

In this section, two adaptive parameter estimation algorithms are presented for constant parameters and time-varying parameters, respectively. The estimation of constant parameters is first presented to show the merit of the suggested new adaptive law. Then this idea is further tailored to address the estimation of time-varying parameters, since some parameters involved in the realistic PEMFC model are time-varying.

For the simplicity of notation and the generality of techniques to be developed, the following nonlinearly parametric system is considered:

$$
y=f(x, u, \theta)
$$

where $x \in \mathbb{R}^{m \times 1}$ is the system state; $u \in \mathbb{R}^{n \times 1}$ is the input; $y \in \mathbb{R}^{q \times 1}$ is system output; $\theta \in \mathbb{R}^{p \times 1}$ represents the unknown parameters to be estimated; and $f(x, u, \theta)$ is the nonlinearly parametric function involving the unknown parameters $\theta$.

Before we present the adaptive parameter estimation algorithm, the following practically feasible assumption is given:

Assumption 1: The nonlinear function $f(x, u, \theta)$ is continuous and differentiable with respect to the unknown parameter $\theta$. The unknown parameter, $\theta$, and its derivative are bounded, (i.e. $\|\dot{\theta}(t)\| \leq \varepsilon$, for a constant $\varepsilon>0$ ).

Remark 1: The above assumption can be trivially fulfilled in the practical operation of PEMFC systems. For instance, the input/output are all bounded, and the operation scenarios allow smooth behaviors involved in $f(x, u, \theta)$. The variation of unknown parameters $\theta$ is also bounded in the realistic PEMFC plants. Moreover, the upper bound $\varepsilon$ of $\dot{\theta}$ is used for analysis only, which is not necessarily known.

As explained in Section II, to address the nonlinearly parametric problem that cannot directly apply real-time adaptive parameter algorithms, we will reformulate system (8) by using the Taylor series expansion with respect to the parameters, which was used in the fuel cell field [25]. Hence, by applying the Taylor series expansion on (8), we have

$$
y=f(x, u, \hat{\theta})+\sum_{i=1}^{p}\left(\theta_{i}-\hat{\theta}_{i}\right) \frac{\partial f(x, u, \hat{\theta})}{\partial \theta_{i}}+\xi(\tilde{\theta})
$$

where $\hat{\theta} \in \mathbb{R}^{p \times 1}$ is the estimated parameters; $\tilde{\theta} \triangleq \theta-\hat{\theta}$ denotes the estimation error; $\xi(\tilde{\theta})$ is the residual error, which stems from the higher-order terms of the Taylor series expansion.

Furthermore, we can rewrite the above equation as

$$
y=g(x, u, \hat{\theta})+F(x, u, \hat{\theta}) \theta+\xi(\tilde{\theta})
$$

where the known regressor matrix $F(x, u, \hat{\theta}) \in \mathbb{R}^{q \times p}$ is calculated by

$$
F(x, u, \hat{\theta})=\left[\begin{array}{ccc}
\left.\frac{\partial f_{1}(x, u, \theta)}{\partial \theta_{1}}\right|_{\theta=\hat{\theta}} & \cdots & \left.\frac{\partial f_{1}(x, u, \theta)}{\partial \theta_{p}}\right|_{\theta=\hat{\theta}} \\
\vdots & \ddots & \vdots \\
\left.\frac{\partial f_{q}(x, u, \theta)}{\partial \theta_{1}}\right|_{\theta=\hat{\theta}} & \cdots & \left.\frac{\partial f_{q}(x, u, \theta)}{\partial \theta_{p}}\right|_{\theta=\hat{\theta}}
\end{array}\right] ;
$$


The known nonlinear vector $g(x, u, \hat{\theta}) \in \mathbb{R}^{q \times 1}$ is expressed as

$$
g(x, u, \hat{\theta})=f(x, u, \hat{\theta})-F(x, u, \hat{\theta}) \hat{\theta}
$$

which can be computed in real-time.

Remark 2: In practical operation, the input, output and state of PEMFC are all measurable and bounded. Thus, the regressor $F(x, u, \hat{\theta})$ is bounded. Moreover, the residual term, $\xi(\tilde{\theta})$, stemming from the high-order Taylor series expansion, is a function of the estimation error $\tilde{\theta}$. Thus, the effect of this residual term can be reduced when the estimation error finally converges to a small compact set. Without loss of generality, we have $\|F\| \leq \eta,\|\xi\| \leq v$ for positive constants $\eta>0$ and $v>0$.

\section{A. Adaptive Estimation for Constant Parameter}

In this subsection, we first present an adaptive estimation method for constant parameter $\theta$ to show the merit of this new estimation algorithm, which serves as the basis of the estimation of time-varying parameters to be presented in the next subsection. In order to obtain the implicit relation between the unknown constant parameter $\theta$ and the measurable variables (i.e. $y, F$, and $g$ ), the filtered auxiliary matrix $M \in \mathbb{R}^{p \times p}$ and vector $n_{1} \in \mathbb{R}^{p \times 1}$ are defined as

$$
\begin{array}{ll}
\dot{M}(t)=-\kappa M(t)+F^{\mathrm{T}} F, & M(0)=0 \\
\dot{n}_{1}(t)=-\kappa n_{1}(t)+F^{\mathrm{T}}(y-g), & n_{1}(0)=0
\end{array}
$$

where $\kappa>0$ is a forgetting factor.

Then, another auxiliary vector $h$ is defined as

$$
h=M \hat{\theta}-n_{1}
$$

This variable $h$ can be online calculated based on the measured input/output data with the regressor $F$ and function $g$, and thus it can be used for the parameter estimation. To show the merit of the induced variable $h$, we have the following Lemma:

Lemma 1: From the auxiliary vector $h(t)$ in (12) based on the filtered matrix $M(t)$ in (10) and the filtered vector $n_{1}(t)$ in (11), we can derive that

$$
h=-M \tilde{\theta}+D_{1}
$$

where $D_{1}(t)=-\int_{0}^{t} e^{-\kappa(t-\tau)} F^{\mathrm{T}}(\tau) \xi(\tau) d \tau$ is a bounded vector (i.e. $\left\|D_{1}\right\| \leq\|F\|\|\xi\| / \kappa \triangleq \sigma_{1}$ ).

Proof: By integrating (10) and (11), we obtain

$$
\left\{\begin{array}{l}
M(t)=\int_{0}^{t} e^{-\kappa(t-\tau)} F^{\mathrm{T}}(\tau) F(\tau) d \tau \\
n_{1}(t)=\int_{0}^{t} e^{-\kappa(t-\tau)} F^{\mathrm{T}}(\tau)(y(\tau)-g(\tau)) d \tau
\end{array}\right.
$$

From (9), we can obtain that $y-g=F \theta+\xi$. Substituting this fact into (14), then $n_{1}=M \theta+D_{1}$ is derived, which together with (12) implies (13).

Remark 3: The constant $\kappa$ in (10) and (11) serves as the forgetting factor to retain the boundedness of the derived variables $M, n_{1}$, and determines the ultimate bound of residual error $D_{1}$. Thus, the constant $\kappa$ should be set as a tradeoff between the convergence rate and the estimation accuracy.

It is shown in Lemma 1 that the obtained variables $h$ contains the estimation error $\tilde{\theta}$, and thus they can be used to design an adaptive law to achieve guaranteed convergence, where the widely used observers or predictors are avoided [14]. On the other hand, it is known that the persistent excitation (PE) condition is necessary in the adaptive parameter estimation [26]. This condition can be fulfilled in the PEMFC plant due to the variations of the voltage. Moreover, this paper will introduce a feasible technique to online test the required excitation condition, which can be summarized in the following lemma:

Lemma 2: Supposing that the regressor matrix $F$ satisfies the PE condition (i.e. $\exists \tau>0$ and $\alpha>0$, such that $\left.\int_{t}^{t+\tau} F^{\mathrm{T}}(\tau) F(\tau) d \tau \geq \alpha I, \forall t>0\right)$, then the derived matrix $M$ in (10) is positive definite (i.e. its minimum eigenvalue $\left.\lambda_{\min }\{M(t)\}>\beta>0, \forall t>0\right)$.

Proof: The proof of Lemma 2 is similar to that shown in our previous work [27]. Thus, it will not be given here due to the limited space.

Now the adaptive law for estimating constant parameter is given as

$$
\dot{\hat{\theta}}=-\Gamma h
$$

where $\Gamma>0$ is a learning gain.

Theorem 1: Consider the system (9) and the adaptive law (15) with filtered matrix (10) and vector (11). Suppose the regressor $F$ satisfies the PE condition, then the estimation error $\tilde{\theta}$ finally converges to a small compact set around zero.

Proof: Selecting $V=\tilde{\theta}^{\mathrm{T}} \Gamma^{-1} \tilde{\theta} / 2$ as the Lyapunov function, then we can evaluate its derivative by substituting $\dot{\tilde{\theta}}=-\dot{\hat{\theta}}$ into (13) and (15), which yields

$$
\dot{V}=\tilde{\theta}^{\mathrm{T}} \Gamma^{-1} \dot{\tilde{\theta}}=-\tilde{\theta}^{\mathrm{T}} M \tilde{\theta}+\tilde{\theta}^{\mathrm{T}} D_{1}
$$

Applying the Young's inequality, i.e. $a^{\mathrm{T}} b \leq a^{\mathrm{T}} a /(2 m)+$ $m b^{\mathrm{T}} b / 2$ for any positive constant $m$, the derivative of $V$ satisfies

$$
\begin{aligned}
\dot{V} & \leq-\left(\beta-\frac{1}{2 m}\right)\|\tilde{\theta}\|^{2}+\frac{m\left\|D_{1}\right\|^{2}}{2} \\
& \leq-\psi_{1} V+\zeta_{1}
\end{aligned}
$$

where $\psi_{1}=2(\beta-1 /(2 m)) / \lambda_{\max }\left\{\Gamma^{-1}\right\}$ and $\zeta_{1}=m \sigma_{1}^{2} / 2$ are all positive constants if we set $m>1 /(2 \beta) ; \lambda_{\max }\left\{\Gamma^{-1}\right\}$ is the maximum eigenvalue of the matrix $\Gamma^{-1}$. Then by further solving the inequality (17), we can obtain that $V(t) \leq$ $e^{-\psi_{1} t} V(0)+\zeta_{1} / \psi_{1}$, which together with the definition of Lyapunov function $V$ gives the norm bound of the estimation error as

$$
\begin{aligned}
\|\tilde{\theta}\| & \leq \sqrt{\frac{2 V}{\lambda_{\min }\left\{\Gamma^{-1}\right\}}} \leq \sqrt{\frac{2 \zeta_{1}+2 \psi_{1} V(0) e^{-\psi_{1} t}}{\psi_{1} \lambda_{\min }\left\{\Gamma^{-1}\right\}}} \\
& \leq \sqrt{\frac{2 \zeta_{1}+\psi_{1} e^{-\psi_{1} t} \lambda_{\max }\left\{\Gamma^{-1}\right\}\|\tilde{\theta}(0)\|^{2}}{\psi_{1} \lambda_{\min }\left\{\Gamma^{-1}\right\}}}
\end{aligned}
$$

where $\lambda_{\min }\left\{\Gamma^{-1}\right\}$ is the minimum eigenvalue of $\Gamma^{-1}$.

\section{B. Adaptive Estimation Method for Time-varying Parameter}

The above adaptive estimation scheme in Section III. A. assumes that the parameters to be estimated are constant, and shows the main idea of the design of adaptive laws by using the extracted parameter estimation error. However, this assumption 
may not be always true for the studied PEMFC system, and the use of constant estimation algorithm may lead to degraded performance (as shown in the subsequent experimental validation). Thus, this subsection presents a further tailored adaptive estimation method for time-varying parameter $\theta(t)$. Following the same procedure of constant parameter estimation, we first define the filtered matrix $M$, which is given in (10). Moreover, a filtered vector $n_{2} \in \mathbb{R}^{p \times 1}$ is defined as

$$
\dot{n}_{2}(t)=-\kappa n_{2}(t)+F^{\mathrm{T}}(y-g-F \hat{\theta})-M \dot{\hat{\theta}}, \quad n_{2}(0)=0
$$

Then, the following auxiliary vector is defined as

$$
h_{1}(t)=F^{\mathrm{T}} F \hat{\theta}-F^{\mathrm{T}}(y-g)
$$

Now, we have the following lemma showing the merits of the above defined variables:

Lemma 3: From the filtered matrix $M(t)$ in (10), the filtered vector $n_{2}(t)$ in (19) and the auxiliary vector $h_{1}(t)$ in (20), we can derive that

$$
\begin{aligned}
& n_{2}=M \tilde{\theta}-D_{2} \\
& h_{1}=-F^{\mathrm{T}} F \tilde{\theta}+F^{\mathrm{T}} \xi
\end{aligned}
$$

where $D_{2}(t)=-\int_{0}^{t} e^{-\kappa(t-\tau)}\left(F^{\mathrm{T}}(\tau) \xi(\tau)-M(\tau) \dot{\theta}(\tau)\right) d \tau$ is a bounded vector (i.e. $\left\|D_{2}\right\| \leq \sigma_{2}$ holds for a constant $\sigma_{2}>0$ ).

Proof: By multiplying $e^{\kappa t}$ on both sides of (19), recalling (14), and the facts $y-g-F \hat{\theta}=F \tilde{\theta}+\xi$ from (9) and $\dot{\hat{\theta}}=\dot{\theta}-\dot{\tilde{\theta}}$, we can obtain

$$
\begin{aligned}
e^{\kappa t} \dot{n}_{2}+\kappa e^{\kappa t} n_{2} & =e^{\kappa t} F^{\mathrm{T}} F \tilde{\theta}+e^{\kappa t} F^{\mathrm{T}} \xi-e^{\kappa t} M \dot{\theta}+e^{\kappa t} M \dot{\tilde{\theta}} \\
& =e^{\kappa t} F^{\mathrm{T}} F \tilde{\theta}+\left(\int_{0}^{t} e^{\kappa \tau} F^{\mathrm{T}}(\tau) F(\tau) d \tau\right) \dot{\tilde{\theta}} \\
& +e^{\kappa t}\left(F^{\mathrm{T}} \xi-M \dot{\theta}\right)
\end{aligned}
$$

Based on (23), it can be rewritten as

$$
\begin{aligned}
\frac{d}{d t}\left(e^{\kappa t} n_{2}\right) & =\frac{d}{d t}\left[\left(\int_{0}^{t} e^{\kappa \tau} F^{\mathrm{T}}(\tau) F(\tau) d \tau\right) \tilde{\theta}\right] \\
& +e^{\kappa t}\left(F^{\mathrm{T}} \xi-M \dot{\theta}\right)
\end{aligned}
$$

By integrating (24), we can derive that

$$
\begin{aligned}
n_{2}(t) & =\left(\int_{0}^{t} e^{-\kappa(t-\tau)} F^{\mathrm{T}}(\tau) F(\tau) d \tau\right) \tilde{\theta} \\
& +\int_{0}^{t} e^{-\kappa(t-\tau)}\left(F^{\mathrm{T}}(\tau) \xi(\tau)-M(\tau) \dot{\theta}(\tau)\right) d \tau
\end{aligned}
$$

Then the fact (21) is verified. Similarly, by substituting (9) into (20), then (22) can be proved.

Compared with the estimation algorithm for constant parameters shown in (15), the proposed variables $h_{1}, n_{2}$ also contain the estimation error $\tilde{\theta}$, and thus they can be used to design an adaptive law to achieve guaranteed convergence. However, since time-varying parameters are considered in this case, specific modifications on $n_{2}$ should be made to derive (21). Moreover, the essential difference between $n_{2}$ and $h_{1}$ lies in that the associated regressors, $M$ and $F^{\mathrm{T}} F$. It is noted in (14) that the matrix $M$ is the integration version of $F^{\mathrm{T}} F$ with penalty coefficient $\kappa$, which introduces an averaging effect. As shown in [14], the use of $h_{1}$ with instant error information is essential for estimating time-varying parameters.

Hence, the adaptive law for estimating time-varying parameter $\theta(t)$ is defined as

$$
\dot{\hat{\theta}}=\Gamma\left(n_{2}-\ell h_{1}\right)
$$

where $\ell>0$ is another learning constant to be tuned.

Now the main results of this subsection are given as follows:

Theorem 2: Consider the system (9) and the adaptive law (26) with filtered matrix (10), and vectors defined in (19)-(20). Suppose the regressor $F$ satisfies the PE condition, then the estimation error $\tilde{\theta}$ converges to a small small compact set.

Proof: Selecting $V=\tilde{\theta}^{\mathrm{T}} \Gamma^{-1} \tilde{\theta} / 2$ as the Lyapunov function, then we can evaluate its derivative by using the fact $\dot{\tilde{\theta}}=\dot{\theta}-\dot{\hat{\theta}}$, and (26) with (21) - (22), such that

$$
\begin{aligned}
\dot{V} & =\tilde{\theta}^{\mathrm{T}} \Gamma^{-1} \dot{\tilde{\theta}}=-\tilde{\theta}^{\mathrm{T}} \Gamma^{-1} \dot{\hat{\theta}}+\tilde{\theta}^{\mathrm{T}} \Gamma^{-1} \dot{\theta} \\
& =-\tilde{\theta}^{\mathrm{T}} M \tilde{\theta}+\tilde{\theta}^{\mathrm{T}} D_{2}-\ell \tilde{\theta}^{\mathrm{T}} F^{\mathrm{T}} F \tilde{\theta}+\ell \tilde{\theta}^{\mathrm{T}} F^{\mathrm{T}} \xi+\tilde{\theta}^{\mathrm{T}} \Gamma^{-1} \dot{\theta}
\end{aligned}
$$

Applying the Young's inequality, the derivative of $V$ satisfies

$$
\begin{aligned}
\dot{V} & \leq-\left(\beta-\frac{3}{2 m}\right)\|\tilde{\theta}\|^{2}+\frac{m \sigma_{2}^{2}}{2}+\frac{m \eta^{2} v^{2} \ell^{2}}{2}+\frac{m \epsilon^{2}}{2 \lambda_{\min }^{2}\{\Gamma\}} \\
& \leq-\psi_{2} V+\zeta_{2}
\end{aligned}
$$

where $\psi_{2}=2(\beta-3 /(2 m)) / \lambda_{\max }\left\{\Gamma^{-1}\right\}$ and $\zeta_{2}=$ $m\left(\eta^{2} v^{2} \ell^{2}+\sigma_{2}^{2}\right) / 2+m \epsilon^{2} /\left(2 \lambda_{\min }^{2}\{\Gamma\}\right)$ are all positive constants if we set $m>3 /(2 \beta)$; Then by solving (28), we can obtain that $V(t) \leq e^{-\psi_{2} t} V(0)+\zeta_{2} / \psi_{2}$. Substituting this solution into the Lyapunov function, the norm bound of estimation error is given by

$$
\begin{aligned}
\|\tilde{\theta}\| & \leq \sqrt{\frac{2 V}{\lambda_{\min }\left\{\Gamma^{-1}\right\}}} \leq \sqrt{\frac{2 \zeta_{2}+2 \psi_{2} V(0) e^{-\psi_{2} t}}{\psi_{2} \lambda_{\min }\left\{\Gamma^{-1}\right\}}} \\
& \leq \sqrt{\frac{2 \zeta_{2}+\psi_{2} e^{-\psi_{2} t} \lambda_{\max }\left\{\Gamma^{-1}\right\}\|\tilde{\theta}(0)\|^{2}}{\psi_{2} \lambda_{\min }\left\{\Gamma^{-1}\right\}}}
\end{aligned}
$$

The proof of Theorem 2 is completed.

The proposed estimation method for time-varying parameters in the PEMFC is shown in Algorithm 1.

Remark 4: From (18) and (29) and the proof of Theorem 1 and Theorem 2, the excitation level $\beta$ and the constant gain $\Gamma$ determine the ultimate size of the estimation error $\tilde{\theta}$. Moreover, the convergence speed also depends on $\beta$ and $\Gamma$. The residual error $\epsilon$ from $\dot{\theta}$ and the term $\xi$ from the Taylor expansion in (29) also affect the size of estimation error $\tilde{\theta}$. Hence, the initial value $\hat{\theta}(0)$ could be properly set to enhance the estimation response.

Remark 5: It is noted that the vector $h$ in (13) and the vector $n_{2}$ in (21) including the estimation error $M \tilde{\theta}$ are employed to drive the adaptive law defined in (15) and (26). Thus, the observers and predictors that are required in most of existing adaptive parameter estimation methods are avoided. Moreover, $h_{1}$ in (22) with the instant information of estimation error is introduced in order to improve the ability of tracking the timevarying behaviors in the unknown parameters. 


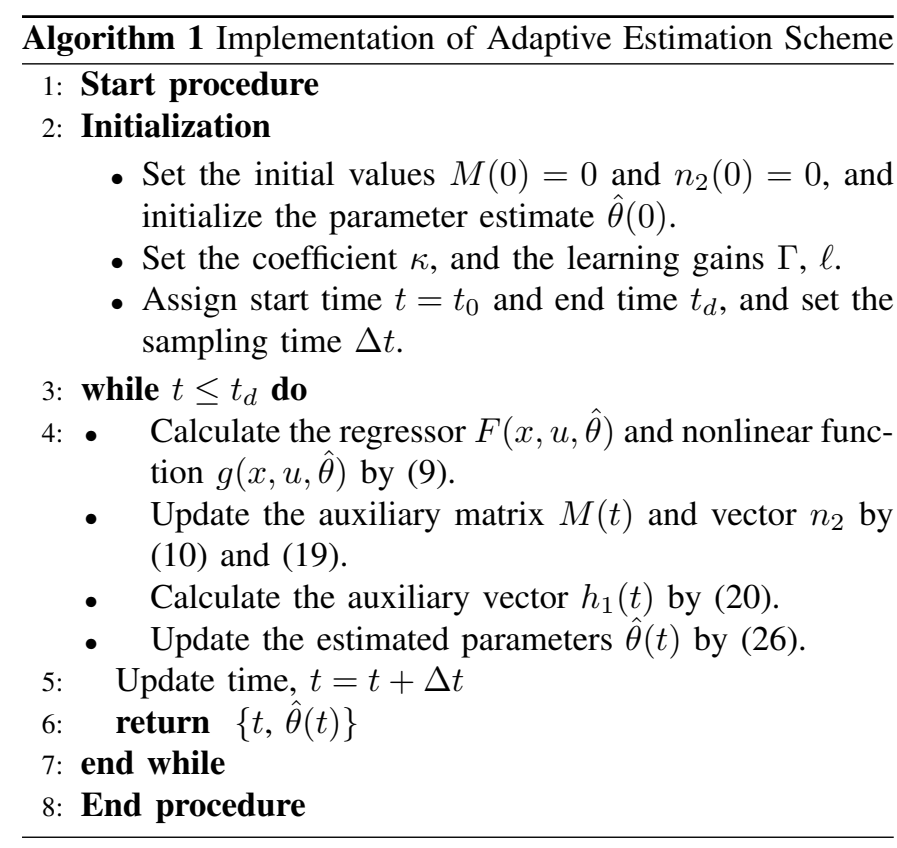

Remark 6: The regressor matrix $F$ should satisfy the PE condition in order to retain the convergence of adaptive laws. This can be fulfilled in our case studies due to the variations in the voltage. Specifically, the online validation of the PE condition is still an open problem, while Lemma 2 provides a feasible method to test the required excitation condition by testing the minimum eigenvalues of the derived matrix $M$. Nevertheless, it deserves further investigation to relax the $\mathrm{PE}$ condition by applying some emerging techniques such as composite learning [28], [29], though this is out of the scope of the current paper.

Remark 7: As shown in the proof of Theorem 1 and Theorem 2, the convergence of the proposed adaptive laws (15) and (26) are proved by using quadratic Lyapunov functions. As shown in [30]-[32], non-quadratic Lyapunov functions may be used to obtain faster convergence rate, which will be studied in our future work.

\section{ExPERIMENTAL SETUP AND MOdel VALIDATION}

This section first introduces the experimental setup of the $\mathrm{H}$ 100 PEMFC system. Then, the mathematical model provided in Section II is also validated in this section.

\section{A. Description of Experimental Setup}

The studied system is an Horizon ${ }^{\circledR} \mathrm{H}-100$ open cathode proton exchange membrane fuel cell system. Figure 2.a. shows a view of this fuel cell device as obtained from the distributor. In particular, the PEMFC stack is a self-humidified fuel cell with 20 cells and an active area of $22.5 \mathrm{~cm}^{2}$ per cell. Thus, the humidify system is not necessary in this system. Figure 3 shows a schematic diagram of the system behavior. A fan controlled through a pulse-width-modulation (PWM) duty cycle signal is used to force the air flow in the fuel cell, feed the cathode with the oxygen required for the reaction, and regulate the PEMFC temperature. The anode is fed with dry hydrogen coming from a hydrogen tank. Moreover, the original H-100

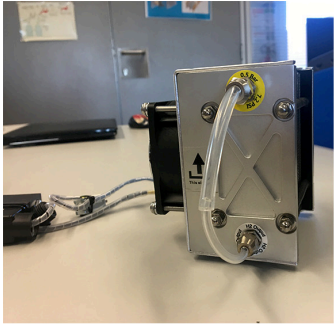

(a)

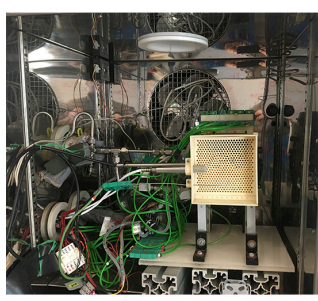

(b)

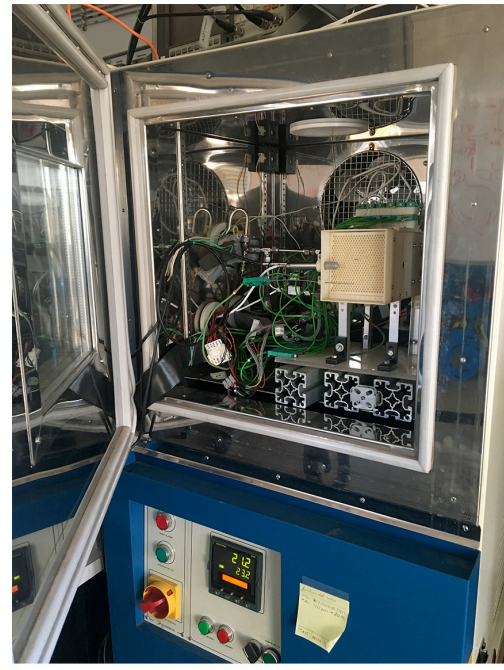

(c)
Figure 2. Laboratory test rig: (a) Commercial H-100 PEMFC; (b) View of the $\mathrm{H}-100 \mathrm{PEMFC}$ in the test rig system; (c) View of the environmental chamber

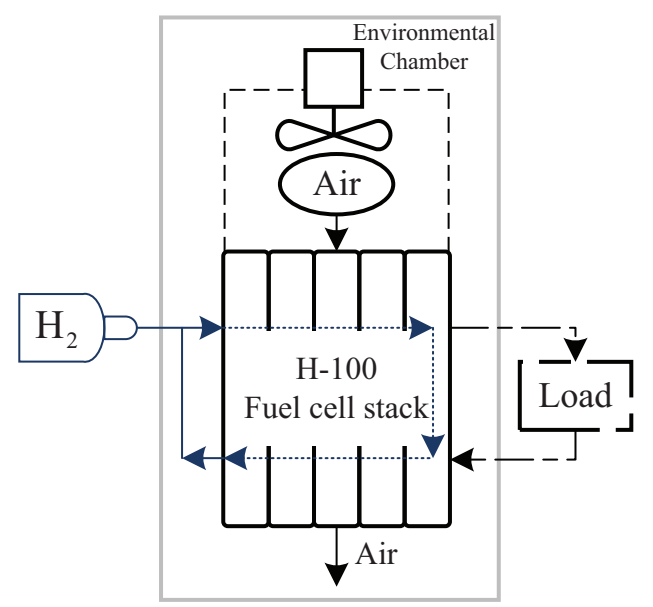

Figure 3. Schematic diagram of the H-100 PEMFC system

PEMFC is embedded in a case, where additional sensors are included (see Figure 2.b). The experiments are carried out inside a climatic chamber to obtain repetitiveness of the experiments (see Figure 2.c).

\section{B. Model Validation}

Before using the parameter estimation algorithms, the PEMFC model described in Section II is validated by comparing its behavior with experimental data. Figure 4. shows the evolution of the fuel cell voltage and temperature when the current is kept constant at $I=3.95 \mathrm{~A}$ and the speed fan changes. It is shown that the model output reproduces the measured variables appropriately. More details of the model validation and experimental conditions can be found in [22].

From the results given in Figure 4, it can be claimed that the derived mathematical model presents a good fitting response for the voltage and temperature, showing the correctness and 


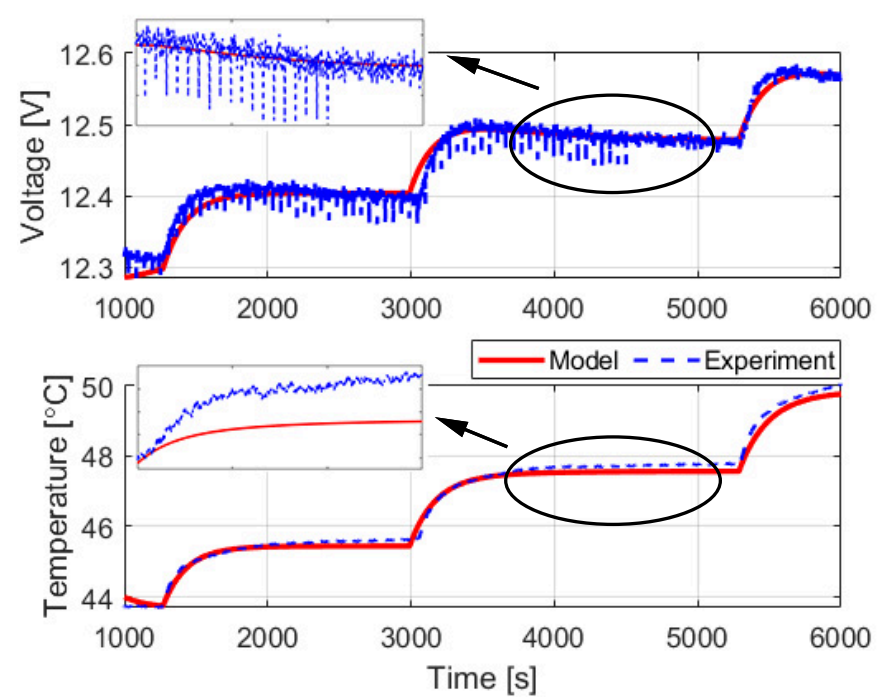

Figure 4. Comparison between the model and the experimental data (Experiment I)

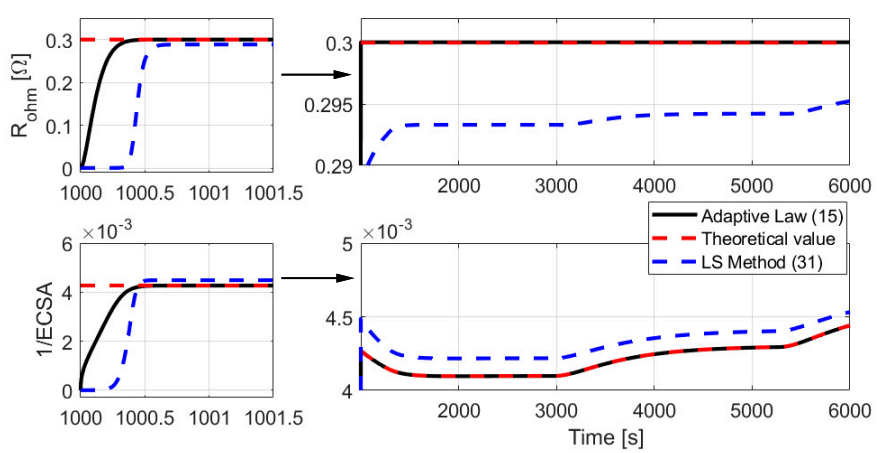

Figure 5. Parameter estimation results for adaptive law (15) and LS approach (30) (Experiment I - model data).

effectiveness of the derived model, though there are certain modeling errors in the voltage.

\section{EXPERIMENTAL RESULTS}

In this section, two experiments are carried out to validate the parameter estimation algorithms. The first one (Experiment I) corresponds to a short experiment where the fuel cell current is kept constant to $I=3.95$ A while the fuel cell temperature changes due to a change in the fan speed. In this experiment the hydrogen is recirculated and the purge mechanism is active to guarantee the enough quantity of hydrogen. In the second one (Experiment II), a longer experiment is performed. In this experiment the current is dynamically changed to trigger time-varying behaviours while the fan speed is kept constant. During this second experiment in a flow-through mode, the purge mechanism is not required.

Table I

COMPARISON OF ESTIMATION PERFORMANCE.

\begin{tabular}{ccc}
\hline Index & Model output error with (26) & Model output error with (15) \\
\hline$I S E$ & 0.0733 & 0.7815 \\
$M A E$ & 1.1823 & 1.2173 \\
$S D$ & 0.0040 & 0.0123 \\
\hline
\end{tabular}
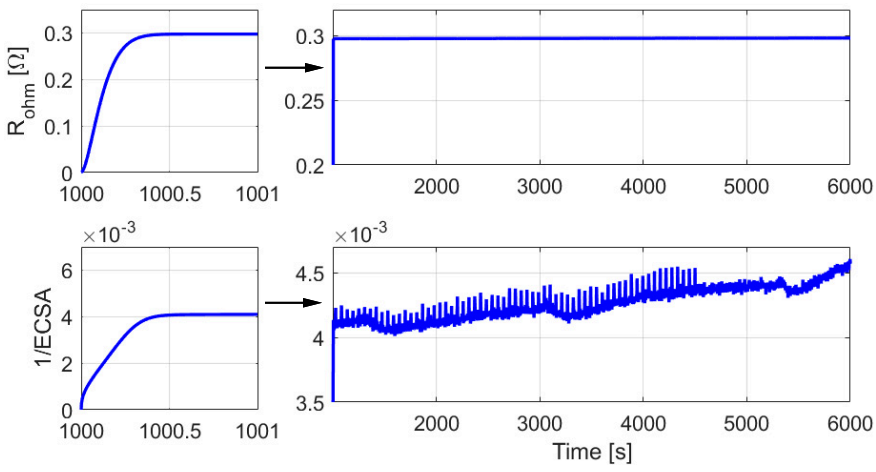

Figure 6. Parameter estimation results for adaptive law (26) (Experiment I experiment data).

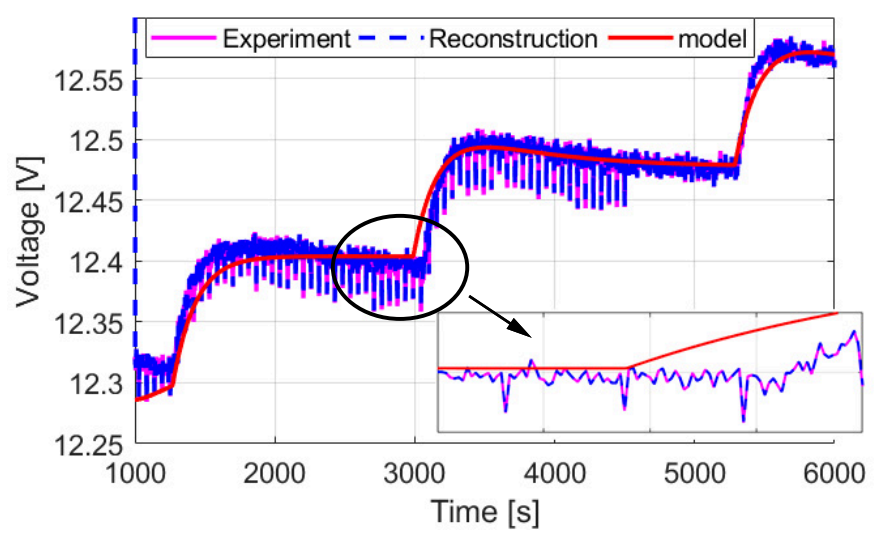

Figure 7. Model output voltage with (15), (26) and the experimental data (Experiment I).

\section{A. Experiment I}

In order to verify the parameter estimation method, we first estimate the parameters using the model data (Section IV). In this case, the nominal parameter values are exactly known. Moreover, the exiting parameter estimation method, LS algorithm [33], is performed for comparison. The LS approach with a variable forgetting factor is given by

$$
\dot{\hat{\theta}}=\frac{P F^{\mathrm{T}} e}{m^{2}}
$$

where the observer error $e$ is deduced as $e=y-\hat{y}=F \tilde{\theta}+\xi$; the designed observer is $\hat{y}=F(x, u, \hat{\theta}) \hat{\theta}+g(x, u, \hat{\theta})$ and the disturbance is bounded (i.e. $\left.\|\xi\| \leq \mu_{0}\right) ; m^{2}=1+\left\|F^{\mathrm{T}} F\right\|$ denotes the normalizing factor; $P$ is a time-varying gain, which is updated by

$$
\dot{P}(t)=-\frac{P F^{\mathrm{T}} F P}{m^{2}}+\delta_{L S}\left(I-\gamma_{L S} P\right) P
$$

where $\delta_{L S}>0$ represents a forgetting constant matrix; The initial value of $P$ satisfies $0<\left(1 / \nu_{L S}\right) I \leq P(0)=P_{0}=$ $P_{0}^{\mathrm{T}}<\left(1 / \gamma_{L S}\right) I ; \nu_{L S}$ and $\gamma_{L S}$ are constants set manually.

For this simulation, the parameters in the proposed method are selected as $\kappa=20$ and $\Gamma=\operatorname{diag}\left(\left[\begin{array}{ll}7 & 14\end{array}\right]\right)$. For the LS method, the parameters are set as $\delta_{L S}=\operatorname{diag}\left(\left[\begin{array}{ll}27 & 10\end{array}\right)\right.$, $\gamma_{L S}=10^{-9}$. The initial values of the estimated parameters are $\theta_{0}=\left[\begin{array}{ll}10^{-3} & 10^{-6}\end{array}\right]^{\mathrm{T}}$. Figure 5. shows the estimation profiles 
of the proposed constant parameter estimator (15) and the LS approach (30). It can be seen that both parameters, $R_{o h m}$ and $1 / E C S A$, converge to the correct value by using the proposed estimator. Moreover, the estimator (15) can achieve faster transient convergence rate than the LS method (30), and the LS method has small steady-state estimation errors as shown in Figure 5.

However, as shown in Figure 4 the derived model with preset parameters $R_{o h m}$ and $1 / E C S A$ cannot accurately capture fast variations on the voltage in the experimental data. In order to obtain accurate values of $R_{o h m}$ and $1 / E C S A$ in Experiment I, the experimental data of temperature and voltage depicted in Figure 4 (blue line) is used as the input for the proposed time-varying estimator (26). The parameters in the simulation are set as $\kappa=20, \ell=0.01$ and $\Gamma=\operatorname{diag}\left(\left[\begin{array}{ll}7 & 14\end{array}\right]\right)$. The profiles of the estimated parameters are depicted in Figure 6. Comparing with the ideal constant values shown in Figure 5, there are slight fluctuations in the two estimated parameters $\left(R_{o h m}, 1 / E C S A\right)$, which stem from the variations in the voltage. Then Figure 7 . shows the reconstructed model output voltages by using the collected temperature data and the estimated parameters (Figure 5) and using the nominal constant parameters (Figure 6). cIt is clearly seen that the reconstructed voltage by using the estimated time-varying parameters provides an overall better model fitting performance in both the steady-state and transient stages, which implies that the fast varying dynamics can be captured with this adaptive law (26) developed for timevarying parameters.

To intuitively show the advantages of the proposed timevarying estimation scheme, the following statistical analysis is used to quantify the model output error performance with the nominal parameters (Figure 5) and the estimated timevarying parameters with adaptive law (26) (Figure 6), respectively. First, the integrated squared error (ISE) represents the performance of overall modeling error, which is calculated as

$$
I S E=\int_{0}^{t} e^{2}(\tau) d \tau
$$

The temporary response of the parameter estimation method can be evaluated by the maximum absolute error (MAE) as

$$
M A E=\max (|e|)
$$

In order to quantify the dispersion of error, the standard deviation (SD) is used, that is

$$
S D=\sqrt{\frac{1}{t} \int_{0}^{t}(e(\tau)-\bar{e})^{2} d \tau}
$$

where $\bar{e}$ is average value of observer error.

The collected voltage and reconstructed model output voltage from $1000 \mathrm{~s}$ to $6000 \mathrm{~s}$ are used to calculate these performance indices. The estimation performance for both estimation methods are summarized in Table I, which indicates that these performance indices of the proposed method are dramatically smaller than that of nominal parameters, especially for ISE.

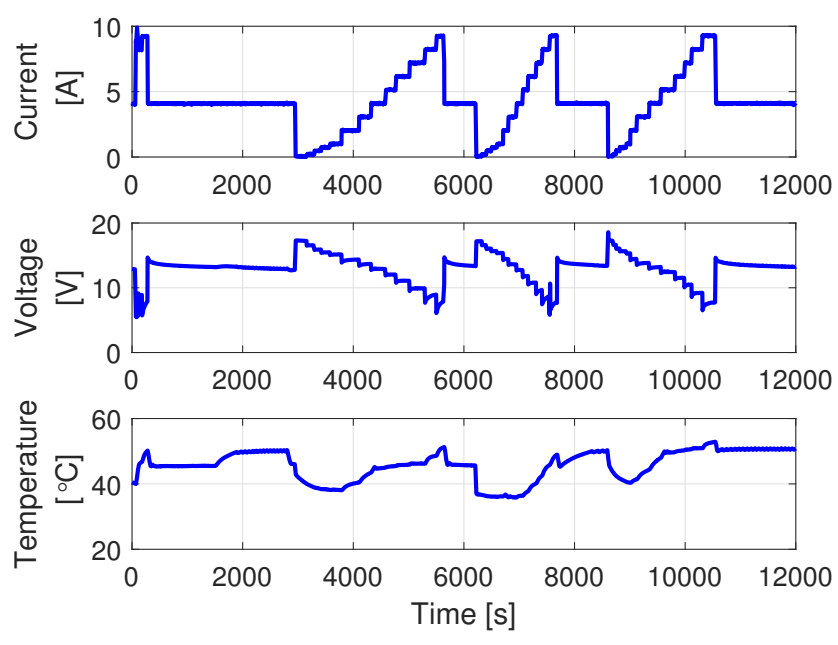

Figure 8. Evolution of fuel cell voltage, temperature and current (Experiment II).

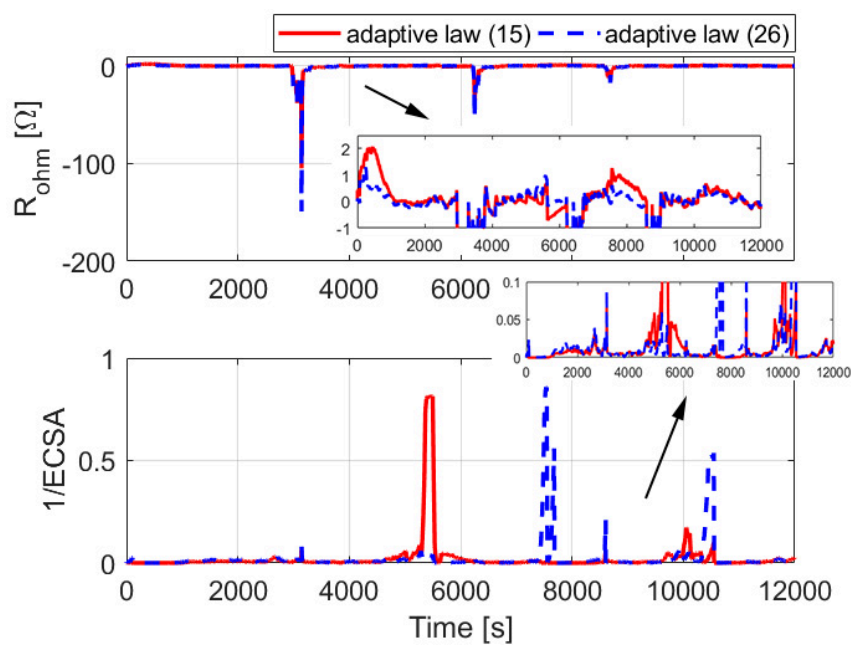

Figure 9. Comparison of the estimated parameters of adaptive law (15) and adaptive law (26) (Experiment II).

\section{B. Experiment II}

This experiment operates a longer time and contains a more dynamic behavior. Figure 8. shows the most relevant variables of PEMFC in this experiment. It will be used to further test the proposed time-varying estimation scheme (26). Hence, the adaptive laws (15) and (26) are used and compared in this case in order to further illustrate the merit of time-varying parameter estimation.

The parameters used in the adaptive law for constant parameter are selected as $\kappa=1, \Gamma=\operatorname{diag}\left(\left[\begin{array}{ll}10^{4} & 55\end{array}\right]\right)$. For time-varying parameter estimation method, the parameters are set as $\kappa=2, \ell=0.05, \Gamma=\operatorname{diag}\left(\left[\begin{array}{ll}10^{4} & 55\end{array}\right]\right)$. Moreover, the initial values of the estimated parameters are set as $\theta_{0}=\left[\begin{array}{ll}10^{-3} & 10^{-6}\end{array}\right]^{\mathrm{T}}$. The parameter estimation results of both adaptive laws (15) and (26) are depicted in Figure 9. The estimated parameter profiles are to some extent similar by using both adaptive laws. However, it is found that the sharp peaks in the parameters can be captured by using the 

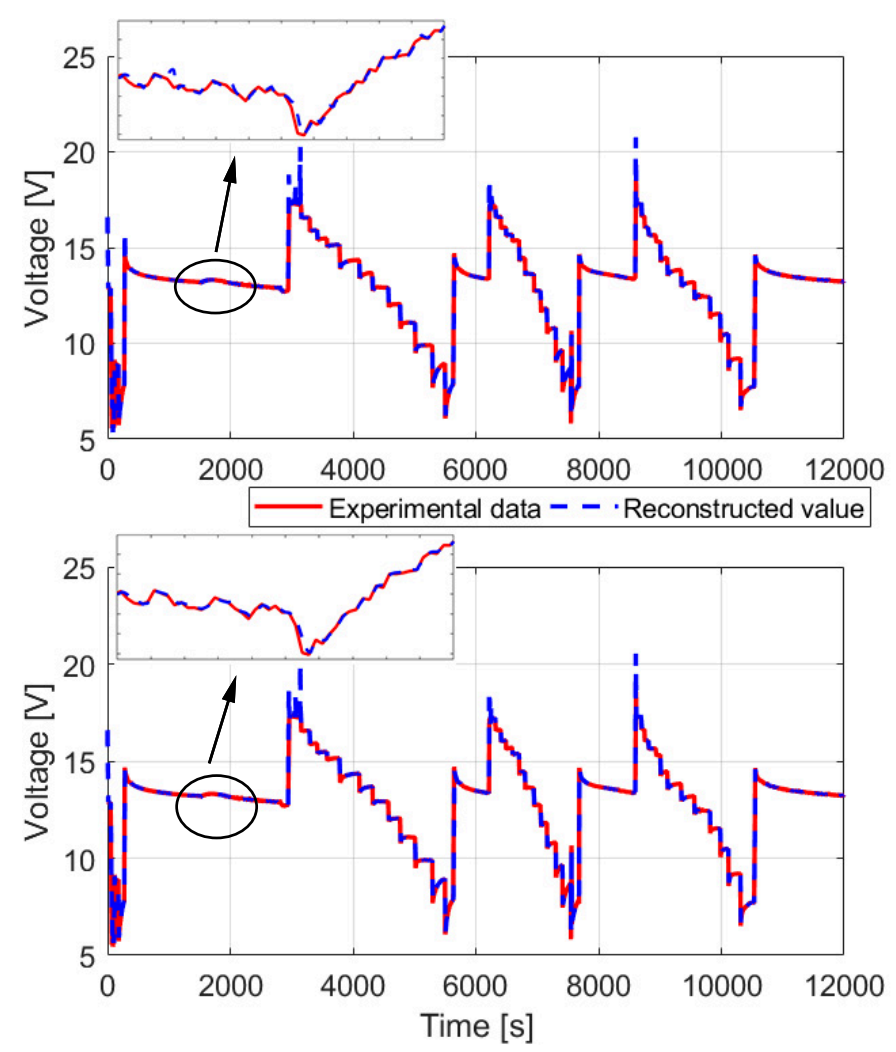

Figure 10. The reconstructed model output voltage results: (a) adaptive law (15); (b) adaptive law (26) (Experiment II).

time-varying estimation scheme (26), which in turn contributes to a better accuracy of PEMFC model. Accordingly, the reconstructed model output voltage profiles with the estimated parameters are shown in Figure 10, which implies that the adaptive laws (15) and (26) can help to obtain a very satisfactory modeling precision. More specifically, it is found that the transient convergence rate of the adaptive law (26) with $h_{1}$ is faster than the adaptive law (15).

In order to show the advantages of time-varying parameter estimation approach, we use the statistical analysis in terms of error indices (32), (33), and (34) to quantify the estimation performance of adaptive laws (15) and (26). The collected voltage and reconstructed model output from $3314 \mathrm{~s}$ to $5176 \mathrm{~s}$ are used to calculate these performance indices. The estimation performance for both adaptive laws are summarized in Table II. The proposed method for time-vary parameter improves the ISE, MAE and SD response, which means an overall better estimation response due to the use of the instant error information in $h_{1}$, beyond the averaged error information in $n_{2}$.

Table II

COMPARISON OF ADAPTIVE LAW PERFORMANCE.

\begin{tabular}{ccc}
\hline Index & Adaptive law (15) & Adaptive law (26) \\
\hline \hline$I S E$ & 1.2923 & 1.1588 \\
$M A E$ & 0.5608 & 0.4697 \\
$S D$ & 0.0429 & 0.0348 \\
\hline
\end{tabular}

\section{CONCLUSION}

In this paper, a new adaptive parameter estimation method has been proposed to estimate essential model parameters in PEMFC with fast time-varying dynamics. The ohmic resistance and the electrochemical active surface area are estimated. To handle the difficulty stemming from the nonlinearly parametric property, the model is linearized by using the Taylor series expansion. Moreover, to address the fast varying parameters, a new adaptive estimation method, independent of the widely used observer/predictor, is suggested. The proposed adaptive laws are directly driven by the parameter estimation error, and thus are able to achieve guaranteed convergence. This new estimation framework allows to online test the required PE condition. Comparative experimental results illustrate the efficacy of the proposed estimation parameter methods and their improved performance. In the future work, we will extend the proposed parameter estimation approach to other kinds of fuel cell systems and relax the required PE condition.

\section{REFERENCES}

[1] F. Barbir, PEM Fuel Cells: Theory and Practice. Academic Press, 2005.

[2] O. Z. Sharaf and M. F. Orhan, "An overview of fuel cell technology: Fundamentals and applications," Renewable and Sustainable Energy Reviews, vol. 32, pp. 810-853, apr 2014.

[3] S. Jomori, N. Nonoyama, and T. Yoshida, "Analysis and modeling of PEMFC degradation: Effect on oxygen transport," Journal of Power Sources, vol. 215, pp. $18-27,2012$.

[4] N. Siegel, M. Ellis, D. Nelson, and M. von Spakovsky, "A twodimensional computational model of a PEMFC with liquid water transport," Journal of Power Sources, vol. 128, no. 2, pp. 173-184, apr 2004.

[5] A. Y. Sendjaja and V. Kariwala, "Decentralized Control of Solid Oxide Fuel Cells," IEEE Transactions on Industrial Informatics, vol. 7, no. 2, pp. 163-170, may 2011.

[6] D. Cheddie and N. Munroe, "Review and comparison of approaches to proton exchange membrane fuel cell modeling," Journal of Power Sources, vol. 147, no. 1-2, pp. 72-84, sep 2005.

[7] G. Napoli, M. Ferraro, F. Sergi, G. Brunaccini, and V. Antonucci, "Data driven models for a PEM fuel cell stack performance prediction," International Journal of Hydrogen Energy, vol. 38, no. 26, pp. 11628 11638 , aug 2013.

[8] L. Pisani, G. Murgia, M. Valentini, and B. D'Aguanno, "A new semiempirical approach to performance curves of polymer electrolyte fuel cells," Journal of Power Sources, vol. 108, no. 1-2, pp. 192-203, jun 2002.

[9] C. Lin, "H-infinity robust adaptive control," Ph.D. dissertation, 1996.

[10] S. Boyd and S. Sastry, "Adaptive control: stability, convergence, and robustness," 1989

[11] R. Hecht-Nielsen, "Kolmogorov's mapping neural network existence theorem," in Proceedings of the IEEE International Conference on Neural Networks III. IEEE Press, 1987, pp. 11-13.

[12] S.-L. Dai, C. Wang, and F. Luo, "Identification and Learning Control of Ocean Surface Ship Using Neural Networks," IEEE Transactions on Industrial Informatics, vol. 8, no. 4, pp. 801-810, nov 2012.

[13] M. Kandidayeni, A. Macias, A. A. Amamou, L. Boulon, and S. Kelouwani, "Comparative Analysis of Two Online Identification Algorithms in a Fuel Cell System," Fuel Cells, may 2018.

[14] J. Na, A. S. Chen, G. Herrmann, R. Burke, and C. Brace, "Vehicle Engine Torque Estimation via Unknown Input Observer and Adaptive Parameter Estimation," IEEE Transactions on Vehicular Technology, vol. 67, no. 1, pp. 409-422, jan 2018.

[15] A. M. Annaswamy, F. P. Skantze, and A.-P. Loh, "Adaptive control of continuous time systems with convex/concave parametrization," Automatica, vol. 34, no. 1, pp. 33-49, jan 1998.

[16] Z.-J. Mo, X.-J. Zhu, L.-Y. Wei, and G.-Y. Cao, "Parameter optimization for a PEMFC model with a hybrid genetic algorithm," International Journal of Energy Research, vol. 30, no. 8, pp. 585-597, jun 2006. 
[17] L. Zhang and N. Wang, "An adaptive RNA genetic algorithm for modeling of proton exchange membrane fuel cells," International Journal of Hydrogen Energy, vol. 38, no. 1, pp. 219-228, jan 2013.

[18] K. Priya, T. Sudhakar Babu, K. Balasubramanian, K. Sathish Kumar, and N. Rajasekar, "A novel approach for fuel cell parameter estimation using simple Genetic Algorithm," Sustainable Energy Technologies and Assessments, vol. 12, pp. 46-52, dec 2015.

[19] Z. L. Gaing, "A particle swarm optimization approach for optimum design of PID controller in AVR system," IEEE Transactions on Energy Conversion, vol. 19, no. 2, pp. 384-391, jun 2004.

[20] M. Ye, X. Wang, and Y. Xu, "Parameter identification for proton exchange membrane fuel cell model using particle swarm optimization," International Journal of Hydrogen Energy, vol. 34, no. 2, pp. 981-989, jan 2009.

[21] C. Restrepo, T. Konjedic, A. Garces, J. Calvente, and R. Giral, "Identification of a Proton-Exchange Membrane Fuel Cell's Model Parameters by Means of an Evolution Strategy," IEEE Transactions on Industrial Informatics, vol. 11, no. 2, pp. 548-559, apr 2015.

[22] S. Strahl, A. Husar, P. Puleston, and J. Riera, "Performance Improvement by Temperature Control of an Open-Cathode PEM Fuel Cell System," Fuel Cells, vol. 14, no. 3, pp. 466-478, jun 2014.

[23] S. Strahl and R. Costa-Castelló, "Model-based analysis for the thermal management of open-cathode proton exchange membrane fuel cell systems concerning efficiency and stability," Journal of Process Control, vol. 47, pp. 201-212, nov 2016.

[24] S. Strahl, A. Husar, and A. A. Franco, "Electrode structure effects on the performance of open-cathode proton exchange membrane fuel cells: A multiscale modeling approach," International Journal of Hydrogen Energy, vol. 39, no. 18, pp. 9752-9767, jun 2014.

[25] M. Lebbal and S. Lecœuche, "Identification and monitoring of a PEM electrolyser based on dynamical modelling," International Journal of Hydrogen Energy, vol. 34, no. 14, pp. 5992-5999, jul 2009.

[26] J. J. E. W. L. Slotine, Applied Nonlinear Control. Prentice Hall, 1991.

[27] J. Na, M. N. Mahyuddin, G. Herrmann, X. Ren, and P. Barber, "Robust adaptive finite-time parameter estimation and control for robotic systems," International Journal of Robust and Nonlinear Control, vol. 25, no. 16, pp. 3045-3071, nov 2015.

[28] K. Guo, Y. Pan, and H. Yu, "Composite Learning Robot Control with Friction Compensation: A Neural Network-Based Approach," IEEE Transactions on Industrial Electronics, pp. 1-1, 2018.

[29] Y. Pan and H. Yu, "Composite Learning From Adaptive Dynamic Surface Control," IEEE Transactions on Automatic Control, vol. 61, no. 9, pp. 2603-2609, sep 2016.

[30] Gang Tao, "Adaptive control systems with L/sup 1+o/ tracking," in Proceedings of 1994 American Control Conference - ACC '94, vol. 2. IEEE, 2005, pp. 1267-1268.

[31] M. Hosseinzadeh and M. J. Yazdanpanah, "Performance enhanced model reference adaptive control through switching non-quadratic Lyapunov functions," Systems and Control Letters, vol. 76, pp. 47-55, feb 2015.

[32] G. Tao, "Model reference adaptive control with L1+ $\alpha$ tracking," International Journal of Control, vol. 64, no. 5, pp. 859-870, jul 1996.

[33] M. de Mathelin and R. Lozano, "Robust adaptive identification of slowly time-varying parameters with bounded disturbances," Automatica, vol. 35, no. 7, pp. 1291-1305, jul 1999.

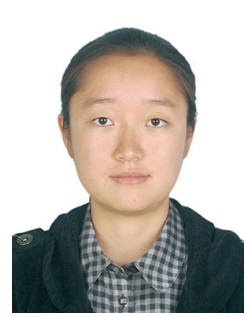

Yashan Xing was born in Yunnan, China. She received the B.Sc. degree in mechanical engineering from Kunming University of Science and Technology, Yunnan, China, in 2014. In 2017, she received the M.Sc. degrees in mechanical engineering from both Blekinge Tekniska Högskola, Blekinge, Sweden and Kunming University of Science and Technology, Yunnan, China. She is currently pursuing the $\mathrm{Ph} . \mathrm{D}$. degree in control engineering from Universitat Politècnica de Catalunya, Barcelona, Spain.

Her current research interests include modelling, adaptive control and parameter estimation for solid oxide fuel cells and solid oxide electrolysis cells

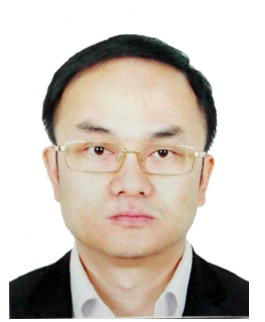

Jing Na (M'15) received the B.Sc. and Ph.D degrees from the School of Automation, Beijing Institute of Technology, Beijing, China, in 2004 and 2010, respectively. From 2011 to 2013, he was a Monaco/ITER Postdoctoral Fellow at the ITER Organization, Saint-Paul-lès-Durance, France. From 2015 to 2017, he was a Marie Curie Intra-European Fellow with the Department of Mechanical Engineering, University of Bristol, U.K.

Since 2010, he has been with the Faculty of Mechanical and Electrical Engineering, Kunming University of Science and Technology, Kunming, China, where he became a Professor in 2013. His current research interests include intelligent control, adaptive parameter estimation, nonlinear control and applications.

$\mathrm{He}$ is currently an Associate Editor of Neurocomputing and has served as an international program committee Chair of ICMIC 2017. Dr Na has been awarded the Best Application Paper Award of the 3rd IFAC International Conference on Intelligent Control and Automation Science (IFAC ICONS 2013), and the 2017 Hsue-shen Tsien Paper Award.

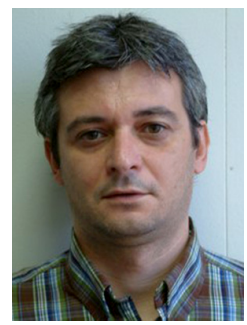

Ramon Costa-Castelló (M'94-SM'07) was born in Lleida, Catalunya, Spain in 1970. He obtained the M.Sc. degree in computer science in 1993 from the Facultat d'Informàtica de Barcelona (FIB), the Universitat Politècnica de Catalunya (UPC). In 2001 he received the $\mathrm{Ph} . \mathrm{D}$. degree in computer science from the Advanced Automation and Robotics (AAR) program from the Cibernetics Institute (Institut de Cibernètica, IC) at UPC.

Currently, he is an Associate Professor at the Automatic Control department (Department of Enginyeria de Sistemes Automàtica i Informàtica Industrial, ESAII) from UPC and the Institut de Robòtica i Informàtica Industrial (a Joint Research Center of the Spanish Council for Scientific Research (CSIC) and UPC).

$\mathrm{He}$ is a Senior Member from the Institute of Electrical and Electronics Engineers (IEEE), member of the Comité Español de Automática (CEA) and member of IFAC (EDCOM, TC 9.4 Committee). 